\title{
Safety and Efficacy of Combined Gluteal Lipofilling with Silicone Cohesive Implants for Gluteal Enhancement in an Egyptian Population
}

\author{
AMR A. EL-NAGGARY, M.D.* and WAEL N.T. AZIZ, M.D.** \\ The Departments of Plastic Surgery, Faculty of Medicine, Fayoum University* and The Department of General Surgery, \\ Faculty of Medicine, Cairo University**
}

\begin{abstract}
Background: Buttock augmentation surgery is on the rise in the recent years. Different approaches exist with different advantages and complication rates. Furthermore, ethnic groups might have different aesthetic demands.

Material and Methods: Here, we describe our experience with a combined lipofilling and silicone implant approach in 20 female Egyptian patients.

Results: Our results reveal both an objective success with an average achieved waist-to-hip ratio of 0.78 after 6 months post-operatively, together with a subjective success with statistically-significantly higher scores reported by both the patients and two independent aesthetic surgeons. Our complication rates were minimal and planned for.

Conclusion: Gluteal lipofilling can thus safely and effectively be combined with silicone implants for gluteal enhancement, giving better shape and projection and avoiding implant dissatisfaction.
\end{abstract}

Keywords: Gluteal enhancement - Buttock fat transfer Gluteoplasty - Implant - Autologous fat transfer.

\section{INTRODUCTION}

Buttock augmentation surgery has been widely on demand in the recent years owing to more focus on body sculpting and the widespread media access to global figures [1]. The ideal waist-to-hip ratio of 0.7 that had been previously regarded as the gold standard may even see a shift in the coming years towards a lower ratio and a curvier figure [2]. Many factors affect the perception of optimum buttock size, including important sociodemographic and cross-cultural ones, but there seems to be a global consensus when it comes to waist-to-hip ratio [3].

The main methods used to achieve buttock augmentation are the introduction of prostheses, autologous fat sculpting, or a mixture of both techniques [4]. The advancement of lipoinjection techniques in the modern times has allowed larger infiltrations in multiple areas [1]. The fat is placed in the gluteal region within the superficial intramuscular or subcutaneous plane with the goal of augmenting fat survival [5]. Contrarily, implant insertion for buttock augmentation (that has doubled in annual numbers between 2005 and 2010) involves one of the 4 anatomical planes: Subcutaneous, subfascial, submuscular, or intramuscular [5]. The subfascial plane is, however, the most preferred position by surgeons [6].

A combined butt augmentation technique with implants and fat transfer is a great option for patients that want the maximum amount of projections to improve their overall shape, and still want to have a natural feel to their buttock [7]. The implant is used to shape the buttock giving it a more rounded, lifted and youthful appearance, while the fat transfer serves two purposes. The first is to sculpt the waist as the liposuction that is needed for the fat harvesting is typically removed from the flanks and abdomen. This is useful in transforming the waist-hip region's shape from square to hourglass. The second use is that fat transfer gives the implant an almost natural appearance with minimal capsular contracture all around [8]. Generally speaking, the more fat there is around the implant (whether it is fat that was naturally there or fat that was transferred), the more buffered the implant is [9].

The above procedure, while optimal, is challenging with variable reported outcomes and complications. Previously reported to have a complication rate of 38.1 percent, a recent systematic review demonstrated a significantly lower rate, with the most common implant-procedure-related complications being dehiscence, seroma, and in- 
fection, and the most common fat-procedure-related complications being seroma, undercorrection, and postoperative pain [10]. No examination of the complications of the combined approach was attempted to date, but it would be assumed to be higher than each technique alone owing to the more steps and manipulation. This necessitates an experienced surgeon with proper knowledge of the anatomical landmarks, proper decisions during patient selection, procedural components, and extent, and proper instrumentation [11].

Ethnic gluteoplasty is emerging as a hot topic in the scene of buttock augmentation, owing to differences in aesthetic ideals and body characteristics of different ethnic groups [7]. Although the Middle East has witnessed a blooming demand when it comes to aesthetic procedures, literature on the subject remains lacking. Only a single study [12] published in 2011 discussed buttock augmentation using the fat transfer technique in 40 female patients in Kuwait. The study combined liposuction from areas around the buttocks and lipoinjection to achieve volume and projection. This combined enhancement and augmentation technique achieved satisfactory results with minimal complications as reported by the author. To the best of our knowledge, the literature does not have any reports on a combined-technique buttock augmentation in the Middle East, nor does it have any report of buttock augmentation of any sort in Egypt-one of the leading countries in aesthetic procedures in the Middle East.

In this work, we describe our experience with twenty Egyptian women that underwent gluteal augmentation over a two-year period. Intramuscular gluteal implants were placed to obtain optimal filling and expansion of small buttocks, and autologous fat grafting was performed above the gluteal thigh sulcus to prevent implant visibility and palpability due to insufficient soft tissue coverage.

\section{MATERIAL AND METHODS}

This prospective non-randomized single-arm clinical study included 20 female patients with hypotrophic gluteal areas who sought surgical consultation for gluteal enhancement between July 2013 and June 2015. All patients aged between 25 and 47 years and had a complaint of deficient flat buttock. All patients underwent a full medical assessment and were medically free. All patients signed an informed consent to be part of our study that contained a separate document for agreement to photographic documentation.
We employed a preoperative survey to have a baseline quantitative measure of aesthetic perception of the patients' buttocks. This included a 1-10 discrete numeric scale in which a score of 1 represented least satisfaction and a score of 10 represented most satisfaction. The pre-operative survey was administered to each patient as well as two independent plastic surgeons that were not involved in the surgeries nor were they aware of the ongoing study. Results from these surveys were tabulated and left to be analyzed post-operatively to avoid any bias during post-operative surveying.

All patients had their waist-to-hip ratio measured pre-operatively, together with a pre-operative assessment for availability of liposuction fat volume. We followed the Mendieta guidelines for gluteal assessment published in 2006 [13]. Sixteen cases were determined to need liposuction from the $\mathrm{V}$ zone, twelve from the upper buttock (point A) or lateral thigh (point B), and nine from the infragluteal fold. All patients were candidates for combined implants and fat transfer to achieve both projection and contouring.

After routine pre-operative labs and an anesthesiology assessment cleared the patients for surgery, preoperative marking were undertaken in the operating theatre with the patients standing. Under general anesthesia, infiltration of the sites of liposuction was carried out with a solution of $0.9 \%$ saline with added adrenaline. A $4 \mathrm{~mm}$ round-tip cannula was used for liposuction and the aspirated fat was collected in sterile suction flasks. Twelve cases underwent liposuction from the abdomen, flanks, and around the buttock area, while 8 cases underwent liposuction from around the buttock area alone. This decision was based on final desired contouring, truncal obesity status, and patients' preferences.

This was followed by bilateral supragluteal incisions following the supragluteal sulcus of wound length approximately 2 inches and intrtamuscular implantation of biconvex cohesive silicone jell implants with a volume of 450-550cc. The collected fat, after blending with an antibiotic solution to combat infection risk, was injected via $60 \mathrm{~mL}$ syringes into the following regions: Over the implants, in the lower gluteal crease, in the lower lateral quadrant of the buttock and lateral hip; all towards the ends of achieving implant coverage and smooth buttock outline. Suction drains were inserted to be removed after 3-5 days post-operatively, and a pressure garment (Elastoplast) was then applied for 15 days. 
Patients were discharged one day following the surgery, and were prescribed post-operative analgesia (Ibuprofen 600mg twice daily) and antibiotic cover (Amoxicillin/Clavulanic Acid 1g twice daily) for the first week post-operatively. The follow-up schedule was 1 week, 1 month, 3 months, and 6 months. The final end point for assessment was considered 6 months post-operatively to give adequate time for the fat to "take" to the tissues. In each follow-up period the patients were assessed for any complications, and for the contouring and distribution of fat in the buttock region. At 6 months post-operatively, the waist-to-hip ratio was measured and the patients and same independent surgeons were surveyed using the same pre-operative scale.

When appropriate, statistical analysis was undertaken using SPSS Version 20 (IBM Analytics, United States). A cut off of 0.05 was considered for statistical significance.

\section{RESULTS}

The mean age of the patients was 31.5 (SD:6.3). The mean waist-to-hip ratio pre-operatively was 1.27 (SD:0.21). There was no statistically significant correlation between the patients' age and their waist-to-hip ratio ( $r=0.14, p=0.437)$. Regarding the pre-operative survey, the average score obtained from the patients for their self-perceived aesthetic level of the buttock region was 3.7 (SD:1.3). The average score obtained from the first independent surgeon was 4.5 (SD:1.1) while that obtained from the second independent surgeon was 3.85 (SD: 0.99). The combined average score for both independent surgeons was 4.17 (SD:1.1). This was not statistically-significantly different when compared to the patient-perceived scores $(p=0.148)$.

Regarding our surgical outcomes, the results of buttock augmentation were immediately visible; however, we did not see our final results until 36 months after the surgery, after the fat had properly "taken" into the tissues. The pre-operative and post-operative (at 6 months) digital photography of two sample cases are presented in Figs. $(1,2)$. Note in the Fig. (1) the successful correction of buttocks ptosis and using the lipofilling to contour the buttocks and cover the implants. Note also the advantage of lateral hip lipofilling in achieving an "all rounded" appearance. Note in Fig. (2) the added volume and contouring using lipofilling in another patient leading to a more projecting buttock based on the patient's desire.

The post-operative complications encountered over the six-month follow-up interval are summa- rized in (Table 1). The most common complication was wound dehiscence that occurred in three of the twenty patients $(15 \%)$, followed by seroma $(n=$ $2,10 \%)$. Two patients (10\%) had fat underfilling that manifested between the first and third months and led in one case to implant exposure; both required an augmentation procedure with the use of stored extra fat from the initial liposuction procedure.

At the study's endpoint (6 months postoperatively), the mean waist-to-hip ratio was 0.78 (SD:0.16). The mean score of self-perceived aesthetic level of the buttock region post-operatively was 7.4 (SD:1.3) in the patients group. The mean post-operative score for the first independent surgeon was 7.25 (SD:1.37) and for the second independent surgeon was 6.8 (SD:1.44). The overall mean score for the independent surgeons was 7 (SD:1.4).

The major variables, measure of change, and statistical significance of such change are depicted in (Table 2). There was a highly statistically significant reduction in waist-to-hip ratio at 6 months post-operatively $(p<0.0001)$. The changes in aesthetic scale score were also highly statistically significant 6 months post-operatively in both the patients' and combined independent surgeons' responses $(p<0.0001)$.

Table (1): Post-operative complications seen in our sample $(n=20)$ over the 6 months follow-up interval.

\begin{tabular}{ll}
\hline Complication & $\mathrm{N}(\%)$ \\
\hline Wound dehiscence & $3(15 \%)$ \\
Seroma & $2(10 \%)$ \\
Undercorrection & $2(10 \%)$ \\
Transient paresthesia & $1(5 \%)$ \\
Prolonged pain & $1(5 \%)$ \\
\hline
\end{tabular}

Table (2): Comparison of the major variables and perception scores pre-operatively and 6 months postoperatively.

\begin{tabular}{llll}
\hline Variable & $\begin{array}{c}\text { Pre- } \\
\text { operative }\end{array}$ & $\begin{array}{c}\text { Post- } \\
\text { operative }\end{array}$ & $p$-value \\
\hline $\begin{array}{l}\text { - Waist-to-hip ratio } \\
\text { (mean } \pm \text { SD) }\end{array}$ & $1.27 \pm 0.21$ & $0.78 \pm 0.16$ & $<0.0001^{*}$ \\
- Aesthetic perception & $3.7 \pm 1.3$ & $7.4 \pm 1.3$ & $<0.0001^{*}$ \\
$\begin{array}{l}\text { scale, patients } \\
\text { (mean } \pm \text { SD) }\end{array}$ & & & \\
- Aesthetic perception & $4.17 \pm 1.1$ & $7 \pm 1.4$ & $<0.0001^{*}$ \\
$\begin{array}{l}\text { scale, surgeons } \\
\text { (mean } \pm \text { SD) }\end{array}$ & & & \\
\hline
\end{tabular}

*: Statistically significant. 

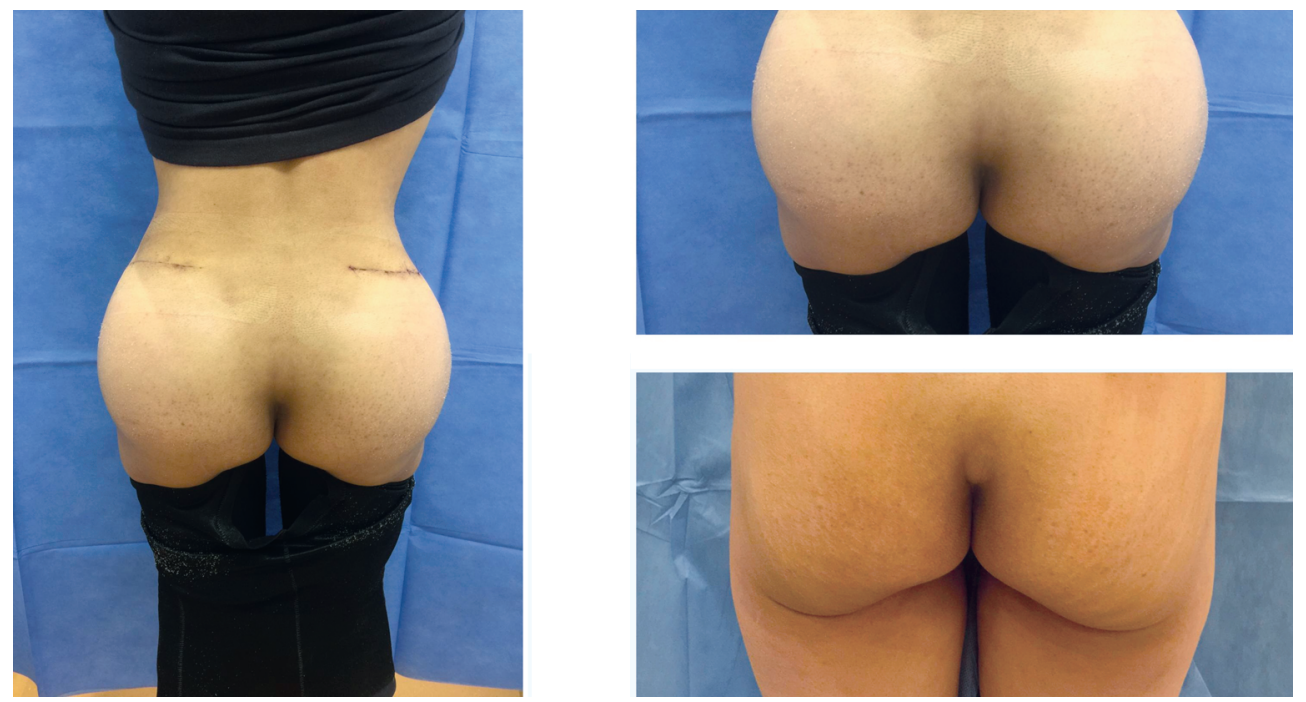

Fig. (1): Pre-operative (Lower right corner) and post-operative (Left side and upper right corner) images of the buttock region of a sample patient as seen from behind. Post-operative image (Upper right corner) is taken at month 6 post-operatively.
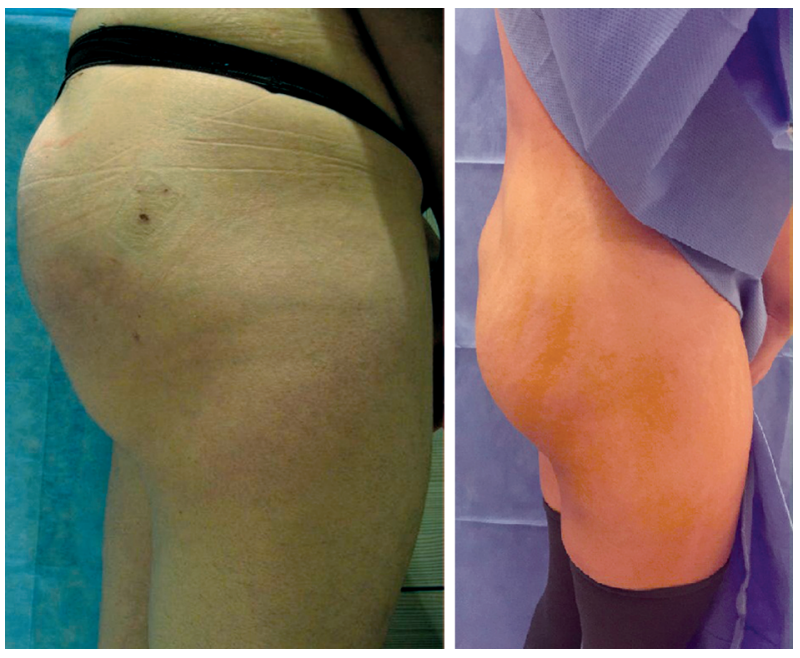

Fig. (2): Pre-operative (Right panel) and postoperative (Left panel) images of the buttock region of a sample patient as seen from lateral view. Post-operative image is taken at month 6 post-operatively.

\section{DISCUSSION}

We presented our experience with buttock augmentation using a combined implant and lipofilling approach in an Egyptian population. Our results reveal that the procedure is both safe and effective, with both subjective (aesthetic scale) and objective (waist-to-hip ratio) measurements revealing statistically significant improvements post-operatively. To the best of our knowledge, this is the first study documenting gluteoplasty cases in an Egyptian population, and the first to assess a combined technique approach in the Middle East.

As the number of gluteoplasty procedures done globally is on the rise [8], it is critical to examine the different approaches to the procedure, and the different ethnic demands [7]. While a global ideal for buttock parameters might exist [2], different populations might have different aesthetic demands and perceptions and should be adequately represented in the literature [14]. A waist-to-hip ratio of 0.7 is considered the global gold-standard [3], and our results do agree with such a statement.

Silicone implants for buttock augmentation were less favored in the literature in a recent systematic review [10]. This might be due to the less invasive and easier technique of day-surgery lipofilling, and not due to its better outcomes. Nevertheless, silicone implants have been reported to achieve better projection, correction of ptosis, and longer stability than lipofilling [8]. Many cases, however, remain unsatisfied following silicone implants majorly due to visibility or palpability of the implant and may require revision surgery [10]. Using an intramuscular implantation approach, this could be avoided by improving upper-pole contour, avoiding edge visibility, having a more stable implant, and reducing muscular dynamics over the implants [15]. This was absolutely true in our experience with intramuscular implantation where none of the cases complained of implant visibility or palpability. This was augmented by fat coverage over the implant edges. Silicone migration is also a recognized complication that should be kept in mind [16], but this usually occurs within the first few months postoperatively, and using an intramuscular approach would be expected to limit its occurrence; it was not encountered in any of our cases. 
Autologous fat grafting for buttock augmentation provides an excellent solution for volume deficiencies, with the combined potential advantage of liposuction from the truncal region [8]. The most serious complications (up to mortality) seem to occur with intramuscular lipoinjection especially in cases when the subcutaneous plane lacks width, but this risk is nearly ameliorated with prior intramuscular implants which acts as a protective barrier $[\mathbf{1 7 , 1 8}]$. Nevertheless, liposculpture is an efficient and promising approach that achieves satisfactory results. A recent study examined 106 consecutive cases that underwent autologous fat grafting and assessed their satisfaction level employing a 4 point scale [4]. The vast majority $(97.1 \%)$ of the cases chose a score of 3 or 4 , which indicated satisfaction. In our approach, we employed a 10 point scale to assess satisfaction levels to give a wider range for subjects' choice and critique. We also employed the opinion of independent aesthetic surgeons to supplement our data. We further opted to have two surveys, one preoperatively and the other at the end point of our study, rather than a single post-operative point assessment. The measure of change in perception is a stronger indicator of satisfaction than a single report of perceived results.

A limitation to our study is that it was a single arm study, and did not compare different techniques of gluteoplasty in different groups of patients to establish superiority of the combined approach. However, many advantages are already reported in the literature of a combined approach over implant-only or lipofilling only approaches [8]. Another limitation is that our follow-up period was only 6 months. The injected fat volume is known to decrease with time, with only $60-70 \%$ of the volume maintained after a year of the procedure [19]. At 6 months, a significant amount of fat volume changes is expected to have occurred, and patients' perception at this end point is considered a great approximate of perception towards final outcomes.

In conclusion, gluteal lipofilling can safely and effectively be combined with silicone implants for gluteal enhancement, giving better shape and projection and avoiding implant dissatisfaction.

\section{REFERENCES}

1- Cárdenas-Camarena L., Arenas-Quintana R. and RoblesCervantes J.A.: Buttocks Fat Grafting: 14 Years of Evolution and Experience. Plast. Reconstr. Surg., Aug., 128 (2): 545-55, 2011.

2- Wong W.W., Motakef S., Lin Y. and Gupta S.C.: Redefining the Ideal Buttocks. Plast. Reconstr. Surg., Jun., 137 (6): 1739-47, 2016.
3- Heidekrueger P.I., Sinno S., Tanna N., Szpalski C., Juran S., Schmauss D., et al.: The Ideal Buttock Size. Plast. Reconstr. Surg., Jul., 140 (1): 20e-32e, 2017.

4- Rosique R.G., Rosique M.J.F. and De Moraes C.G.: Gluteoplasty with Autologous Fat Tissue. Plast. Reconstr. Surg., May, 135 (5): 1381-9, 2015.

5- Cuenca-Guerra R. and Lugo-Beltran I.: Beautiful Buttocks: Characteristics and Surgical Techniques. Clin. Plast. Surg., Jul., 33 (3): 321-32, 2006.

6- Lancerotto L., Chin M.S., Freniere B., Lujan-Hernandez J.R., Li Q., Vasquez A.V., et al.: Mechanisms of Action of External Volume Expansion Devices. Plast. Reconstr. Surg., Sep., 132 (3): 569-78, 2013.

7- Oranges C.M., Gohritz A., Kalbermatten D.F. and Schaefer D.J.: Ethnic Gluteoplasty. Plast. Reconstr. Surg., Oct., 138 (4): 783e-784e, 2016.

8- Oranges C.M., Tremp M., Di Summa P.G., Haug M., Kalbermatten D.F., Harder Y., et al.: Gluteal Augmentation Techniques: A Comprehensive Literature Review. Aesthetic Surg. J., May, 37 (5): 560-9, 2017.

9- Murillo W.L.: Buttock augmentation: Case studies of fat injection monitored by magnetic resonance imaging. Plast. Reconstr. Surg., Nov., 114 (6): 1606-14-6, 2004.

10- Sinno S., Chang J.B., Brownstone N.D., Saadeh P.B. and Wall S.: Determining the Safety and Efficacy of Gluteal Augmentation. Plast. Reconstr. Surg., Apr., 137 (4): 11516, 2016 .

11- Villanueva N.L., Del Vecchio D.A., Afrooz P.N., Carboy J.A. and Rohrich R.J.: Staying Safe during Gluteal Fat Transplantation. Plast. Reconstr. Surg., Jan., 141 (1): 79 86, 2018.

12- Ali A.: Contouring of the Gluteal Region in Women. Ann. Plast. Surg., Sep., 67 (3): 209-14, 2011.

13- Mendieta C.G.: Classification System for Gluteal Evaluation. Clin. Plast. Surg., Jul., 33 (3): 333-46, 2006.

14- Lee E.I., Roberts T.L. and Bruner T.W.: Ethnic considerations in buttock aesthetics. Semin. Plast. Surg., Aug., 23 (3): 232-43, 2009.

15- Godoy P.M. and Munhoz A.M.: Intramuscular Gluteal Augmentation with Implants Associated with Immediate Fat Grafting. Clin. Plast. Surg., Apr., 45 (2): 203-15, 2018.

16- Biguria R. and Ziegler O.R.: Silicone Migration after Buttock Augmentation. Plast. Reconstr. Surgery Glob. Open, Dec., 5 (12): e1583, 2017.

17- Cárdenas-Camarena L., Bayter J.E., Aguirre-Serrano H. and Cuenca-Pardo J.: Deaths Caused by Gluteal Lipoinjection. Plast. Reconstr. Surg., Jul., 136 (1): 58-66, 2015.

18- Mofid M.M., Teitelbaum S., Suissa D., Ramirez-Montañana A., Astarita D.C., Mendieta C., et al. Report on Mortality from Gluteal Fat Grafting: Recommendations from the ASERF Task Force. Aesthetic Surg. J., Jul. 1, 37 (7): 796806, 2017.

19- Swanson E.: Prospective Controlled Study of Buttock Fat Transfer Using Ultrasound and Photographic Measurements. Plast. Reconstr. Surgery Glob. Open, May 4, 4 (5): e697-e697, 2016. 